\title{
Rapid pathology of lumpectomy margins with open-top light-sheet (OTLS) microscopy
}

\author{
Ye Chen, ${ }^{1,5}$ Weisi Xie, ${ }^{1,5}$ Adam K. Glaser, ${ }^{1}$ Nicholas P. Reder, ${ }^{2}$ \\ Chenyi Mao, ${ }^{3}$ Suzanne M. Dintzis, ${ }^{2}$ Joshua C. Vaughan, ${ }^{3,4}$ and \\ JONATHAN T. C. LIU ${ }^{1,2, *}$ \\ ${ }^{1}$ Department of Mechanical Engineering, University of Washington, Seattle, WA 98195, USA \\ ${ }^{2}$ Department of Pathology, University of Washington School of Medicine, Seattle, WA 98195, USA \\ ${ }^{3}$ Department of Chemistry, University of Washington Seattle, WA 98195, USA \\ ${ }^{4}$ Department of Physiology and Biophysics, University of Washington, Seattle, WA 98195, USA \\ ${ }^{5}$ These authors contributed equally \\ *jonliu@uw.edu
}

\begin{abstract}
Open-top light-sheet microscopy is a technique that can potentially enable rapid $e x$ vivo inspection of large tissue surfaces and volumes. Here, we have optimized an open-top light-sheet (OTLS) microscope and image-processing workflow for the comprehensive examination of surgical margin surfaces, and have also developed a novel fluorescent analog of H\&E staining that is robust for staining fresh unfixed tissues. Our tissue-staining method can be achieved within 2.5 minutes followed by OTLS microscopy of lumpectomy surfaces at a rate of up to $1.5 \mathrm{~cm}^{2} /$ minute. An image atlas is presented to show that OTLS image quality surpasses that of intraoperative frozen sectioning and can approximate that of gold-standard H\&E histology of formalin-fixed paraffin-embedded (FFPE) tissues. Qualitative evidence indicates that these intraoperative methods do not interfere with downstream post-operative H\&E histology and immunohistochemistry. These results should facilitate the translation of OTLS microscopy for intraoperative guidance of lumpectomy and other surgical oncology procedures.
\end{abstract}

(c) 2019 Optical Society of America under the terms of the OSA Open Access Publishing Agreement

\section{Introduction}

Approximately 200,000 women in the United States are diagnosed with early-stage invasive breast cancer or carcinoma in situ each year, for which breast-conserving surgery (BCS), or lumpectomy, has become the preferred first-line treatment [1,2]. Numerous clinical studies have shown that there is no difference in overall survival or disease-free survival between patients treated with radical mastectomy vs. BCS with postoperative radiation when complete tumor resection is achieved $[3,4]$. However, a significant challenge for lumpectomy is to ensure that the tumor is completely resected, which is highly correlated with the rate of local recurrence $[5,6]$. Unfortunately, recent studies have reported that $20-40 \%$ of lumpectomy patients require additional surgery due to post-operative histopathology revealing that the resection margins are positive for carcinoma [7-9].

Surgically excised specimens submitted for post-operative histopathological examination are first grossly "bread-loafed" into 3- to 5-mm thick serial slices, from which grossly suspicious regions are selected for further processing into formalin-fixed paraffin-embedded (FFPE) blocks [10]. A 4- $\mu$ m-thick histology section is typically cut from each tissue block and mounted on a glass slide such that each slide displays a cross-section of the specimen from the margin surface down towards the core of the sample [10]. Although slide-based histology provides a clinical gold-standard for margin assessment, it is a time-consuming and laborintensive process [11-15]. Sampling errors are severe due to the selective imaging of only a few thin tissue cross-sections from a large specimen, in which $<1 \%$ of the surgical margin surface is visualized [11-15]. In summary, there is a need for an intraoperative technology to 
enable a comprehensive, non-destructive assessment of lumpectomy margins, which would significantly reduce the costs and inconvenience associated with re-excision surgeries and would also improve patient outcomes by reducing the likelihood of local recurrence [11-15].

As a rapid intraoperative alternative to slow post-operative histology, frozen section histology may be performed to guide certain tumor-removal procedures $[7,16,17]$. However, frozen section histology is not ideal for breast tissues due to their high lipid content, which leads to significant artifacts [16-21]. In addition, because of the technical challenges of preparing adequate numbers of frozen sections within an acceptable intraoperative time frame ( $<30$ minutes), frozen section histology suffers from more-severe sampling limitations than post-operative slide-based histology [19-21]. Finally, frozen sectioning leads to tissue destruction that can negatively affect the accuracy of post-operative archival pathology [1921]. Alternative technologies for nondestructive intraoperative lumpectomy guidance include touch preparation cytology [22,23], ultrasound [24-26], specimen radiography [27,28], radiofrequency spectroscopy [29,30], optical coherence tomography [31], photoacoustics [32], confocal/nonlinear microscopy [33-35], structured illumination microscopy (SIM) [36-38], microscopy with UV surface excitation (MUSE) [39-43], and molecular imaging with topically applied agents [44-47]. Although most of these methods are in various stages of research development or clinical adoption (such as ultrasound and specimen X-ray), the reliability of these tools typically falls short of gold-standard histology due to various factors such as limited spatial resolution, surface sampling, and contrast $[28,29,48]$.

Of these methods, optical-sectioning microscopy offers the potential for robust and rapid microscopic inspection of lumpectomy margins that approaches the image quality of goldstandard histology without requiring destructive physical sectioning [30]. While early systems and studies suffered from limited fields of view $(<1 \times 1 \mathrm{~mm})$ [49-52], in recent decades, widearea imaging of large surgically excised specimens has been achieved with a variety of opticalsectioning techniques such as confocal microscopy [33,53-57], nonlinear microscopy [34,58,59], SIM [37,38,60], and MUSE [39-43]. Confocal and nonlinear microscopy systems tend to be slow since they often require a tightly focused laser beam to be raster scanned in two-dimensions (2D) over a large tissue surface. SIM and MUSE can rapidly capture 2D images of tissue surfaces with a detector array and are relatively cost-effective technologies compared with confocal and nonlinear microscopy. However, image contrast is dependent upon the axial optical-sectioning thickness of these technologies, which is tunable with SIM but tissue-dependent with MUSE ( $\sim 10$ to $25 \mu \mathrm{m}$ according to previous reports [39]). An additional technical constraint of all of these optical imaging systems is that high-numerical-aperture (NA) optics are necessary to achieve sub-nuclear resolution (1-2 $\mu \mathrm{m})$, but this results in a narrow depth of focus $(<5 \mu \mathrm{m})$. A narrow depth of focus can be an impediment for rapid imaging of freshly excised tissue specimens, in which surface irregularities can cause the surgical margin to come in and out of focus [61-64]. In addition, it is difficult in practice to align large tissue surfaces to be perfectly parallel with the focal plane of the microscope during scanned imaging [61-64], or to deal with tissue debris that can obscure the margin surface [39]. These challenges may ultimately hamper the clinical translation of these techniques.

In an attempt to overcome some of the technical challenges encountered by previous microscopy systems, we have developed an open-top light-sheet (OTLS) microscopy system for rapid, wide-area, microscopic imaging of large clinical specimens [65]. In general, lightsheet microscopy achieves optical sectioning by employing thin "selective" illumination of tissues and orthogonal collection of fluorescence signal [66-71]. However, previous light-sheet microscopy systems have been designed to image small model organisms rather than to accommodate large specimens of arbitrary geometry [66-74]. For the OTLS system utilized in this study, tissue specimens are simply placed on a flat glass-plate sample holder without elaborate tissue-mounting protocols (i.e. a "flat-bed scanner" for tissues). A solid immersion lens (SIL) provides wavefront matching of an illumination and collection beam as they transition from air into the higher-index material of the SIL (fused silica, $\mathrm{n} \sim 1.46$ ). The beams 
are then index matched into the tissue sample through a thin oil film $(\mathrm{n} \sim 1.46)$, followed by a fused silica plate $(\mathrm{n} \sim 1.46)$ on which the sample is placed. Altogether, this wavefront- and index-matching strategy allows for aberration-free imaging (Fig. 1) [65]. This unique open-top configuration is convenient for clinical use with essentially no constraints on specimen geometry. An initial publication demonstrated the basic feasibility of achieving rapid intraoperative pathology of the surfaces of freshly excised specimens with OTLS microscopy, using a single-channel acridine orange stain [65]. This initial study also showcased a fluorescent analog of H\&E staining for fixed biopsy samples, consisting of DRAQ5 as a fluorescent analog of hematoxylin (excited at $660 \mathrm{~nm}$ ) and eosin [65], which naturally fluoresces under 488-nm excitation. While this "D\&E" staining method allowed for dualchannel fluorescence imaging that could be false-colored to mimic gold-standard H\&E-stained tissue sections $[65,75]$, it only performed well for staining fixed, dehydrated samples. Our group and others have found that when staining fresh, hydrated specimens, eosin leaks out of the tissue during imaging due to poor binding to tissue structures $[58,59,65,75]$, which creates an unwanted background signal that limits image contrast.

In this study, we have optimized the speed, image-processing, and reliability of OTLS microscopy, and developed an improved two-color fluorescent analog of H\&E staining to enable robust intraoperative pathology of lumpectomy margins. We show that our H\&E analog provides sufficient fluorescence signal to achieve OTLS microscopy at high imaging speed (1.5 $\mathrm{cm}^{2} /$ minute), and that it eliminates the eosin-leaking problem of the previous "D\&E" approach, which thereby enables OTLS images to be obtained with superior image contrast. An image atlas is presented to show that these methods provide surface images that can approximate the quality of gold-standard H\&E histology of FFPE tissues. In addition, a preliminary qualitative comparison of histology results (H\&E histology and immunohistochemistry (IHC)) from adjacent tissue surfaces that have either been processed for OTLS microscopy, or are untouched by our OTLS methods (control tissue), suggests that our tissue-staining and imaging methods do not interfere with downstream post-operative H\&E histology and IHC analysis. These results will facilitate the translation of OTLS microscopy for intraoperative guidance of lumpectomy and other surgical oncology procedures.

\section{Methods}

\subsection{Tissue preparation}

To demonstrate rapid pathology of human breast tissues with an open-top light-sheet (OTLS) microscope, discarded surgically excised breast specimens were obtained fresh from patients undergoing lumpectomies and mastectomies at the University of Washington (UW) medical center, under informed consent. All human tissues were collected and de-identified by the NorthWest BioTrust (NWBT), a tissue banking resource at the UW, with approval by the UW Human Subjects Division.

Fresh surgically excised breast specimens were obtained for our imaging experiments immediately after surgery. After inking all exterior surfaces, each specimen was bisected such that the two adjacent cut-surfaces were mirror images of each other. One half of the specimen (control specimen) underwent routine histological evaluation (H\&E histology and IHC), including formalin fixation and paraffin embedding (FFPE). The other half (experimental specimen) was stained with SYBR Gold and ATTO 655 N-hydroxysuccinimide ester (NHS ester) followed by OTLS microscopy (for $<30$ minutes). After OTLS microscopy, the specimen was submitted for the same histological evaluation performed on its counterpart. As shown in Fig. 2, this study enabled two comparisons: 1) the OTLS surface images were compared to gold-standard H\&E histological images to demonstrate that the image quality of our technique can approximate that of archival H\&E histology of FFPE tissues; 2) the histology results (H\&E and IHC) from experimental specimens (used for OTLS microscopy) were compared to the histology results from corresponding control specimens (untouched by OTLS microscopy 
methods) to show that our staining and imaging methods do not interfere with routine postoperative $\mathrm{H} \& \mathrm{E}$ histology and IHC analysis.

\subsection{An innovative two-color fluorescent analog of H\&E staining}

In this study, SYBR Gold $\left(\lambda_{\mathrm{Ex}} / \mathrm{Em}=495 / 537\right.$, Thermo Fisher, Cat. No: S11494) was utilized as the nuclear stain while ATTO 655 NHS ester $\left(\lambda_{E x} / E m=655 / 680\right.$, Sigma-Aldrich, Cat. No: 76245) was utilized as an eosin-mimicking cytoplasmic/stromal stain. SYBR Gold labels dsDNA, ssDNA and RNA in both fresh and fixed tissues [76]. NHS esters are widely-used amine-reactive functional groups for covalent labeling of primary amines [77] and are commonly used to conjugate antibodies to fluorophores and nanoparticles [78-81]. We hypothesized that an NHS-ester-functionalized fluorophore could be used as a more robust fluorescent analog to eosin, which also stains cytoplasmic proteins indiscriminately, but tends to leach out due to weak noncovalent binding. An additional benefit of this approach is that since many commercially available fluorophores are available as NHS esters, it is straightforward to select fluorophores that avoid spectral cross-talk for dual-channel fluorescence imaging, such as SYBR Gold and ATTO 655.

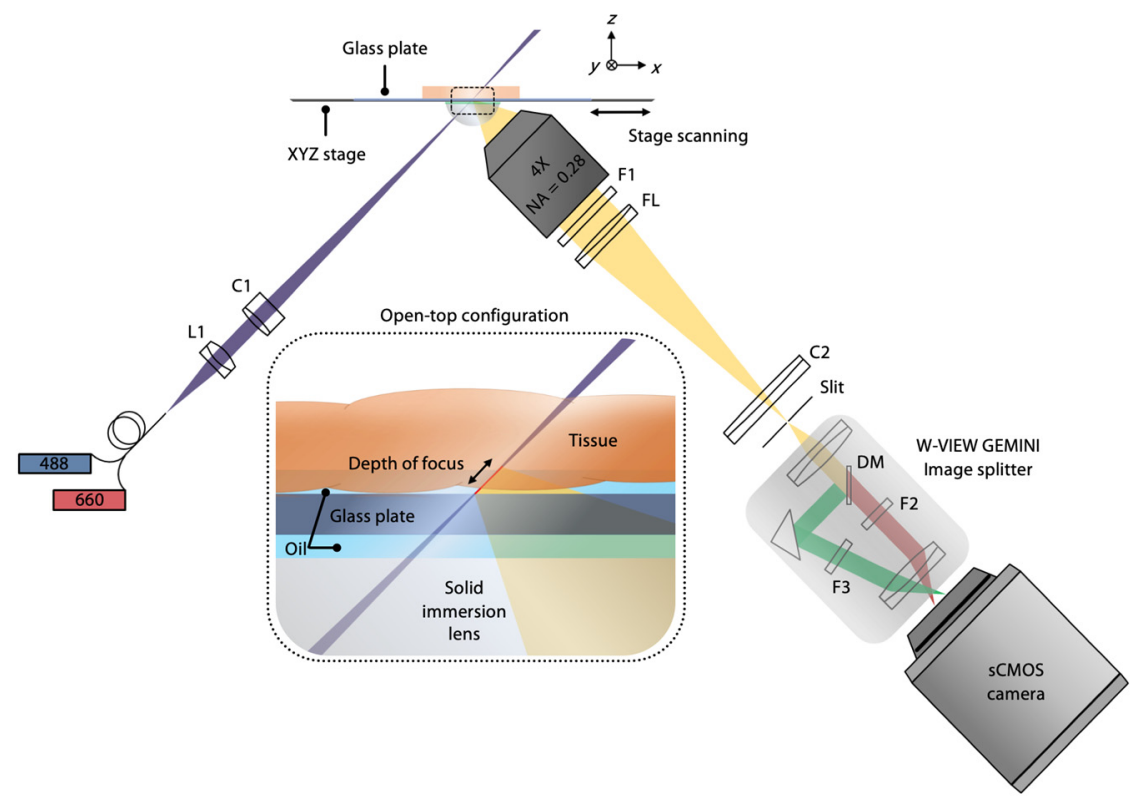

Fig. 1. Schematic of an open-top light-sheet (OTLS) microscope. The OTLS microscope utilizes a solid immersion lens (SIL) and thin oil film to provide wavefront- and index-matching of the illumination and collection beam paths into tissue at a 45-deg angle of incidence. This unique open-top configuration (inset) is versatile for imaging diverse clinical specimens with minimal constraints on size and geometry. The 0.03 illumination NA provides an extended depth of focus $(\sim 400 \mu \mathrm{m})$ to accommodate for tissue-surface irregularities, specimen tilt, and tissue debris.

The following parameters were optimized to achieve high-contrast OTLS images of lumpectomy margins with the SYBR Gold and ATTO 655 NHS ester stain: (1) pH of the staining solution (from $\mathrm{pH}=6.5$ to $\mathrm{pH}=9.5$ ), which is known to affect the reactivity of NHS esters [77]; (2) staining concentration (from $100 \mathrm{nM}$ to $10 \mu \mathrm{M}$ ), which can affect imaging sensitivity/speed; and (3) rinsing duration (from $30 \mathrm{sec}$ to $120 \mathrm{sec}$ ), which can affect image contrast (signal-to-background ratio). As a control experiment, hydrolyzed (inactivated) ATTO 655 NHS ester was applied to tissue surfaces to verify that the image contrast provided by reactive (non-hydrolyzed) ATTO 655 NHS ester was due to covalent labeling rather than noncovalent binding of the dye to the specimen. In this study, our optimized 2.5-minute tissuestaining and rinsing protocol utilized a cocktail containing a 1:15,000 v/v dilution of SYBR 
Gold and $1 \mu \mathrm{M}$ ATTO 655 NHS ester in $1 \times$ Phosphate-Buffered Saline (PBS, Gibco, Cat. No: $10010023)$ at $\mathrm{pH} 8.0$ to stain fresh tissue surfaces for 1 minute, followed by three 30 -sec rinse steps in $1 \times$ PBS at $\mathrm{pH}$ 7.4.

\subsection{An OTLS microscope optimized for rapid dual-channel fluorescence imaging}

An optical schematic of the OTLS system is shown in Fig. 1. Illumination is provided by two fiber-coupled diode lasers (Coherent, OBIS ${ }^{\mathrm{TM}}$ Laser System) at $488 \mathrm{~nm}$ and $660 \mathrm{~nm}$, which are combined into one single-mode optical fiber $\left(1 / \mathrm{e}^{2}\right.$ focusing $\mathrm{NA}=0.12$, Core Diameter $=2.5$ $\mu \mathrm{m})$ and collimated using lens, $L 1(f=25 \mathrm{~mm})$. The collimated beam transmits through a cylindrical lens, $C 1$ (NA =0.03), creating a 2-mm-wide light-sheet $\left(1 / \mathrm{e}^{2}\right)$ with a full-width at half maximum (FWHM) thickness of $\sim 7 \mu \mathrm{m}$ at the beam waist. This is the diffraction-limited FWHM resolution in the worst dimension (along the collection axis) of the OTLS microscope at $660 \mathrm{~nm}$. The 0.03 illumination NA provides the OTLS system with a depth-of-focus (confocal parameter, $\left.2 Z_{R}\right)$ of $\sim 400 \mu \mathrm{m}$. The long depth-of-focus $(\sim 400 \mu \mathrm{m})$ provides our OTLS microscope with the versatility to image diverse clinical specimens that have various degrees of pliability and surface irregularity.

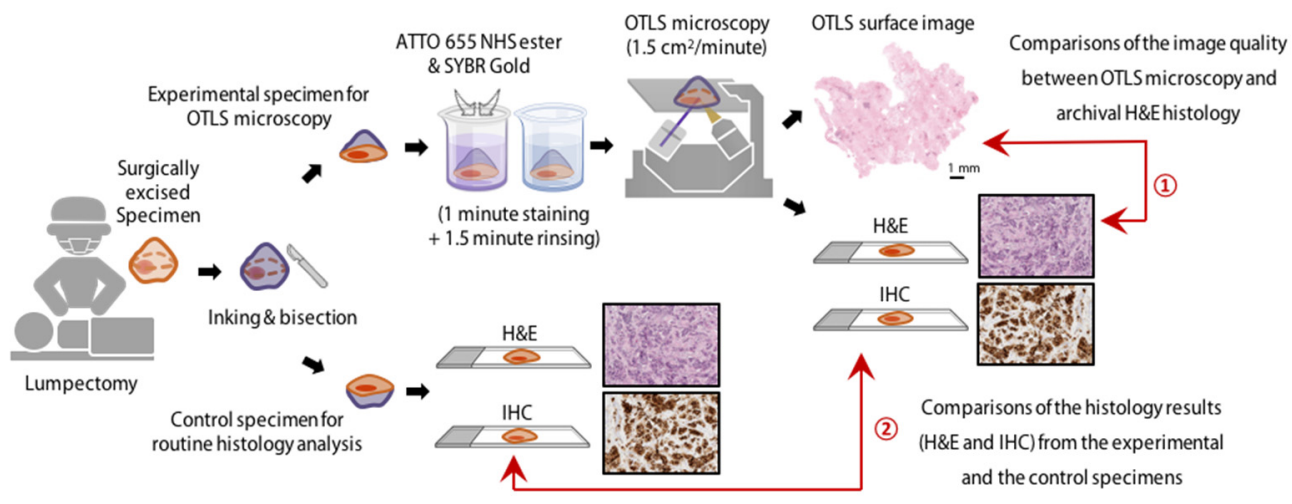

Fig. 2. Study design. Freshly excised human breast tissues were inked and bisected immediately after lumpectomy procedures. The bisected surface from one half of the specimen (control specimen) was processed for routine histology (H\&E and IHC). The bisected surface from the other half (experimental specimen) was stained and imaged with OTLS microscopy $(<30$ minutes), before being processed for routine histology. OTLS surface images were compared to archival H\&E histology. In addition, histology images from the experimental and control specimens were compared to show that our tissue-staining and imaging techniques do not interfere with downstream H\&E histology and IHC.

The OTLS system is equipped with a novel specimen interface [65] (Fig. 1 inset), which is comprised of a 7.5-mm-radius fused-silica solid immersion lens (SIL, BMW Optics, $n=1.46$ ), a 0.5 -mm thick oil layer (Cargille Labs, Cat.No:19571), and a 1-mm-thick fused-silica glass plate, which serves as the sample holder (ESCO Optics, $10 \times 10 \mathrm{~cm}^{2}$ ). When performing scanned imaging of the specimen, the thin oil layer at the top surface of the SIL, which does not evaporate, provides an index-matched interface between the stationary SIL and the horizontally translated glass-plate sample holder. Both the illumination and collection beams are positioned below the glass-plate sample holder, allowing the OTLS microscope to accommodate human tissues of arbitrary size and thickness without physically interfering with the illumination and collection optics. The custom SIL (with a radius of $7.5 \mathrm{~mm}$ ) is truncated with a reduced center thickness of $6 \mathrm{~mm}$ such that the beam waist (optimal focus) of both light beams is located right above the glass plate. Tissue specimens are placed on the glass-plate sample holder along with a thin layer of index-matching oil to prevent the specimens from drying out and also to improve the index matching between the glass plate and the tissue surface. 
Fluorescence emission is collected using a $4 \times$ objective lens, $L 2\left(f_{L 2}=43.47 \mathrm{~mm}, \mathrm{NA}=\right.$ 0.28 ), filtered through an emission filter, F1 (Semrock, FF01-496/LP-25), and focused using a tube lens, $F L\left(f_{F L}=150 \mathrm{~mm}\right)$. A dual-channel image-splitting module (Hamamatsu, W-VIEW GEMINI) is used to focus the two fluorescent channels onto two halves of a high-speed scientific complementary metal-oxide-semiconductor (sCMOS) camera chip (PCO, pco.edge 4.2). To avoid spectral cross-talk between the two image channels (SYBR Gold and ATTO 655 NHS ester), the image-splitting module contains a dichroic mirror, DM (Semrock, Di03-R594t1-25 $\times 36$ ), and two separate emission filters, $F 2$ and $F 3$ (Semrock, FF01-685/LP-25 and SP01561RU-25). A low-power cylindrical lens, $C 2(f=2000 \mathrm{~mm})$ is placed before the camera to provide final correction for astigmatism induced by a slight refractive-index mismatch at the oil-tissue interface. There is a total of $5 \times$ magnification between the specimen and the camera $\left(1.46 \times f_{F L} / f_{L 2}=5\right)$, in which the SIL provides an additional magnification factor that is equivalent to its refractive index of $\sim 1.46$ [82].

The collection objective (Olympus, XLFLUOR $4 \times, \mathrm{NA}=0.28$ ) provides a practical field of view of $\sim 1.25 \mathrm{~mm}$ and a measured lateral resolution (within the plane of the illumination light-sheet) of $\sim 2.0 \mu \mathrm{m}$. As the illumination light sheet is scanned through the sample, a series of dual-channel light-sheet images are captured at an oblique 45-degree angle with an image width, $w$, and adjustable image height, $h$ (Figs. 3(a) and 3(b)). The image width is limited by the 1.25 -mm field of view of the detection objective (Fig. 3(b)). The combined dual-channel image height (Fig. 3(a)) recorded by the camera is 128 vertical camera pixels tall (64 pixels per channel, which is $\sim 80 \mu \mathrm{m}$ in tissues). This reduced crop size is chosen to maximize the imaging speed while still accommodating for any tissue irregularities/tilt/debris at the surfaces of the lumpectomy specimens. As shown in Fig. 3(b), the distance between images along the scan dimension, $x$, is set as $1.25 \mu \mathrm{m}$, which matches the sampling density of the sCMOS camera in the $y$ and $h$ directions ( $1.25 \mu \mathrm{m}$ per pixel).

\subsection{Optimization of the image acquisition and processing pipeline}

Adjacent volumetric OTLS image strips (1.25-mm wide), as shown in Fig. 3(b), are acquired in succession (with 1-mm spacing) using a serpentine scanning pattern to cover the entire surface of each specimen up to a maximum size of $\sim 10 \times 10 \mathrm{~cm}^{2}$, using a high-speed motorized stage (Thorlabs, MLS-203-1). For a raw image size of 1000 pixels (width) by 128 pixels (height), along with a 500- $\mu$ s camera exposure time, the highest imaging speed of the system is $\sim 1.5 \mathrm{~cm}^{2} /$ minute. In this study, the dual-channel raw image frames (1000 pixels by 128 pixels in 16-bit TIFF formats) were acquired at a streaming rate of 1000 pixels $\times 128$ pixels $\times 2.0$ Byte $\times 2000 \mathrm{fps}=0.51 \mathrm{~GB} / \mathrm{s}$ to a dedicated workstation containing a high-speed Redundant Array of Independent Disks (RAID) 0 controller with a PCI CameraLink interface. To synchronize the stage-scanning mechanism with the image acquisition, a custom LABVIEW program was developed.

In an initial LABVIEW design, raw image frames (1000 pixels by 128 pixels) were first buffered to the computer memory as a $1 \mathrm{D}$ array (with a total of 128,000 pixels). This $1 \mathrm{D}$ array was then reshaped into a 2D array for real-time image display. However, when using the NI Vision Development Module (NI-IMAQ Write File) to write each 2D array to disk as a TIFF file, the write speed was limited to $\sim 0.25 \mathrm{~GB} / \mathrm{s}$ due to the overhead involved in creating, opening, and closing each TIFF file. This slow process was not suitable for time-limited applications, such as intraoperative guidance. To overcome this limitation, the Technical Data Management Streaming (TDMS) file format (National Instruments) is utilized for high-speed data streaming in LABVIEW. In summary, after buffering each raw light-sheet image to the computer memory as a $1 \mathrm{D}$ array and reshaping this buffer into a $2 \mathrm{D}$ array for real-time image display, consecutive 2D arrays are concatenated into a pre-initialized TDMS 3D data cube. At the end of each volumetric image strip ( $x$-direction scan of the sample with respect to the light sheet, as shown in Fig. 3(b)), this TDMS file (3D data cube) can be saved to disk all at once, which allows us to maintain the full data streaming speed of $0.51 \mathrm{~GB} / \mathrm{s}$. 
Our light-sheet images are obtained at an oblique 45-deg angle of incidence with respect to the specimen surface. However, when stored digitally, these images are arranged as a rectangular data cube. Therefore, to restore the correct tissue geometry, the data cubes must be sheared by $45 \mathrm{deg}$ in the $\mathrm{x}-\mathrm{z}$ plane to create a trapezoidal data volume (Fig. 3(c)). A custom Python code [83] was written to convert the TDMS file into TIFF images as well as to perform the image shearing.
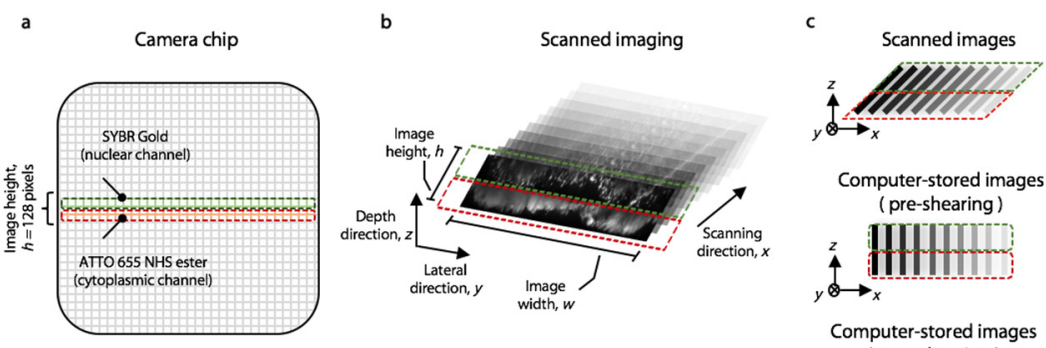

Computer-stored images

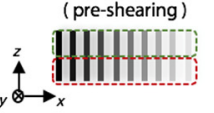

Computer-stored images (post-shearing)
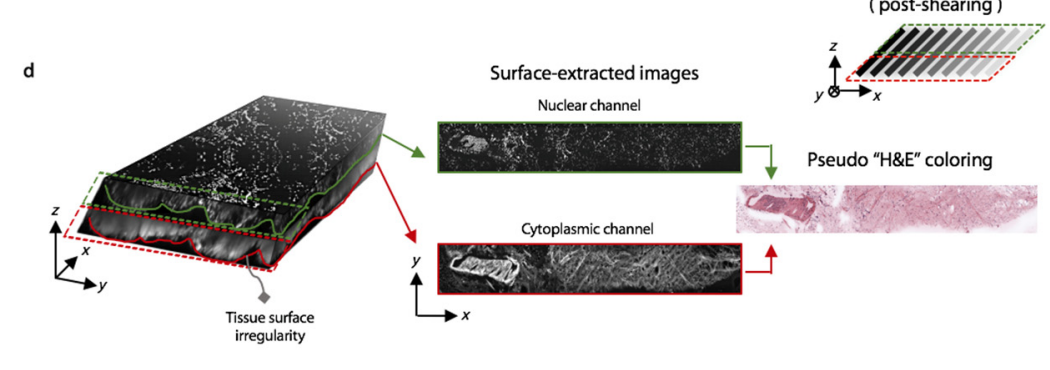

e

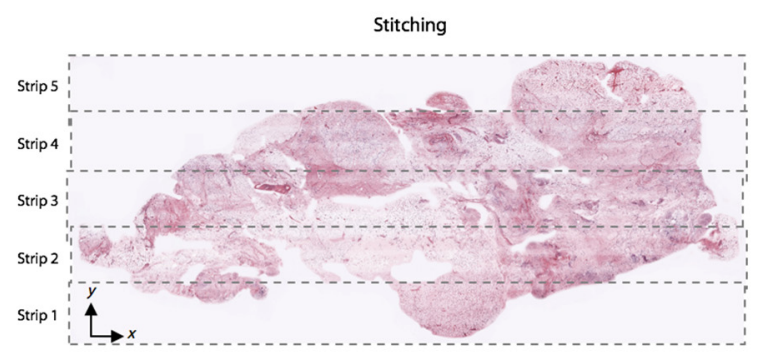

Fig. 3. Image acquisition and processing of OTLS microscopy. (a) The dual-channel (nuclear and cytoplasmic channel) OTLS images occupy a combined height $(h)$ of 128 vertical camera pixels (64 pixels per channel, or $\sim 80 \mu \mathrm{m}$ in tissues). The image height, $h=128$ pixels, was selected to optimize the imaging speed while accommodating for surface irregularities, specimen tilt, and tissue debris. (b) Oblique (45-deg) light-sheet images are captured in succession at a sampling pitch of $1.25 \mu \mathrm{m}$ along the primary tissue-scanning direction, $x$. The horizontal dimension of each image strip is $w=1.25 \mathrm{~mm}$. (c) The raw light-sheet images are initially stored in a rectangular data cube. During post-processing, this data cube is sheared by 45 deg in the $x$ $z$ plane to transform the data cube into a trapezoidal data volume, which accurately represents the geometry of the imaged tissue volume. (d) An extended-depth-of-field (EDF) algorithm [84] is applied to extract the irregular surface of the specimen. The two-channel surface-extracted image is then false-colored to resemble H\&E histology using an algorithm modified from a recent publication [86]. (e) After false-color processing, adjacent image strips are registered and stitched using an ImageJ grid-stitching algorithm [87].

After shearing, an extended-depth of field (EDF) algorithm [84] is applied in ImageJ to the cytoplasmic channel, which exhibits more-complete surface staining compared with the nuclear channel, to extract the "best-focus" surface (i.e. tissue surface) from each 3D image strip. Once the "best-focus" surface has been defined, both of nuclear and cytoplasmic channels at this surface are then used in a false-coloring algorithm to mimic H\&E histology. This custom falsecoloring code [85] is a modified version of an algorithm described in a recent publication [86] (Fig. 3(d)). Adjacent pseudo-colored image strips are then registered and stitched to adjacent 
image strips using the ImageJ grid-stitching algorithm [87] (Fig. 3(e)). The total image processing time (from image-shearing to false-coloring) for a 1-cm by 1-cm sample ( $20.4 \mathrm{~GB}$ of data) is $\sim 15 \mathrm{~min}$. Since this is not ideal for time-limited clinical applications, we are working on optimizing the code, including the use of GPUs (see Discussion and conclusion). It should also be noted that if the "best-focus" surface of the tissue is defined prior to image processing, only a few minutes is required to fully process the OTLS images at one imaging depth.

\section{Results}

We have developed a two-color fluorescent analog of H\&E for staining fresh breast tissues, and have optimized the speed, image-processing, and reliability of our OTLS microscopy system (see Methods for details). By combining our novel tissue-staining method with an improved light-sheet imaging system, we demonstrate the ability to achieve intraoperative pathology of lumpectomy margins that approximates the image quality of slide-based $\mathrm{H} \& \mathrm{E}$ histology without interfering with the current gold standards of H\&E histology and IHC (Fig. 2).

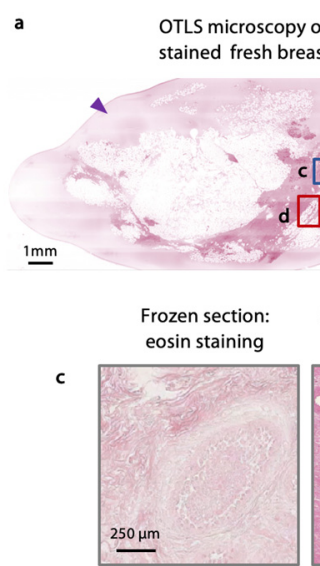

d

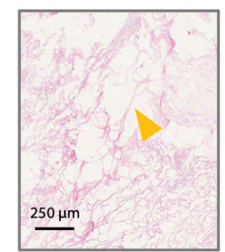

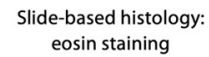
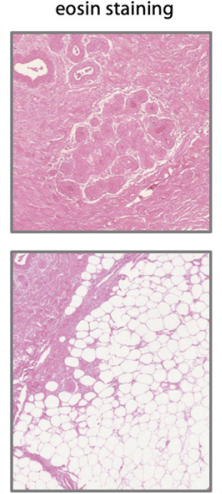

b

OTLS microscopy of ATTO 655 NHS

ester-stained fresh breast tissue

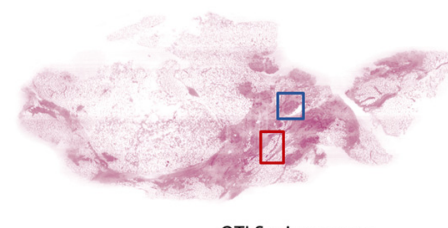

OTLS microscopy: eosin staining

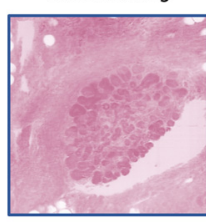

ATTO 655 NHS ester staining
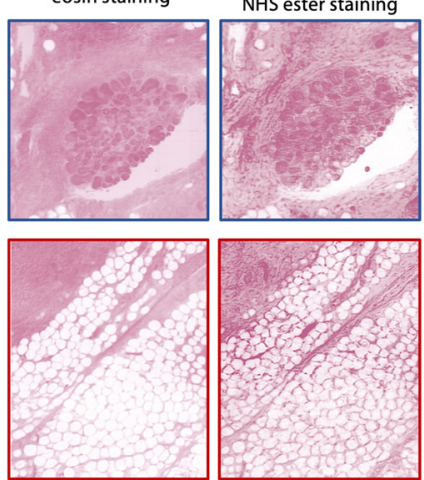

Fig. 4. A comparison of the image quality between OTLS microscopy of eosin-stained (a) and ATTO 655 NHS-ester-stained (b) fresh breast tissue surfaces. When staining fresh specimens, eosin is not stably bound within the tissue and leaks out of the tissue during imaging (purple arrow), which generates a high background that deteriorates the image contrast. The image panels in (c) and (d) provide image quality comparisons between frozen-section histology with eosin staining, archival FFPE histology with eosin staining, OTLS microscopy with eosin staining, and OTLS microscopy with ATTO 655 NHS-ester staining. Results show that the ATTO 655 NHS ester provides improved contrast for visualizing tissue structures, such as collagen fibers, in comparison to eosin in fresh unfixed tissues. Adipocytes and strands of fibrous tissue with stromal cells remained intact after OTLS microscopy and slide-based "H\&E" histology. However, the same tissue structures are heavily distorted in frozen-section "H\&E" histology ((d), yellow arrow).

Figure 4 displays a comparison of the image quality between OTLS microscopy of eosinstained vs. ATTO 655 NHS-ester-stained fresh breast tissues. The purple arrowhead in Fig. 4(a) indicates that eosin is weakly bound within fresh, unfixed breast tissues, which causes the eosin to leak out even after multiple rinse steps. This phenomenon has been seen in other reports in the past $[58,59,65,75]$. Various tissue structures selected from Figs. 4(a) and 4(b) are compared 
to similar tissue structures from both frozen-section and FFPE histology with eosin staining (Figs. 4(c) and 4(d)). Inspection of selected micro-architectural features indicates that the ATTO 655 NHS ester stain provides higher-contrast visualization of specific structures, such as collagen fibers, than eosin does in fresh, hydrated specimens. Finally, it is apparent that nondestructive OTLS microscopy preserves the architecture of the fibroadipose tissue, in a manner comparable to archival FFPE histology, whereas underlying architecture of the breast tissue is heavily distorted in frozen-section histology (yellow arrowhead).

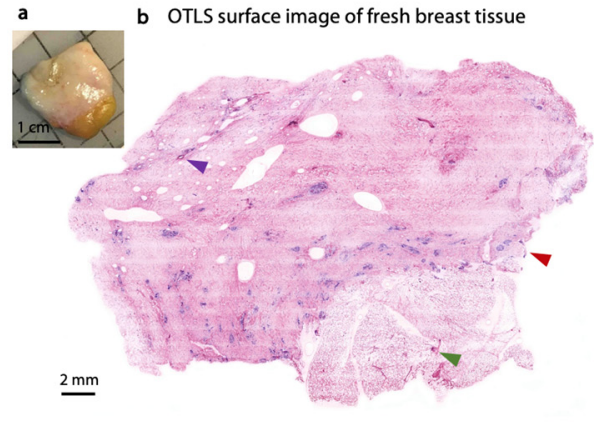

d OTLS microscopy
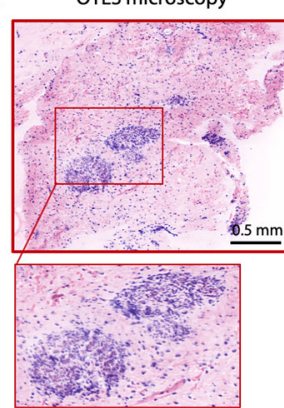

Slide-based H\&E

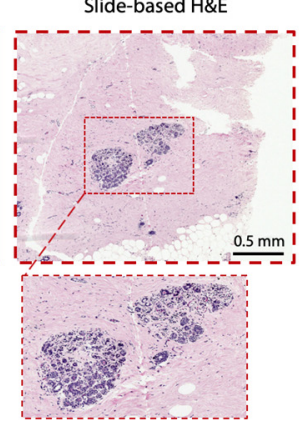

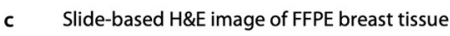

c Slide-based H\&E image of FFPE breast tissue

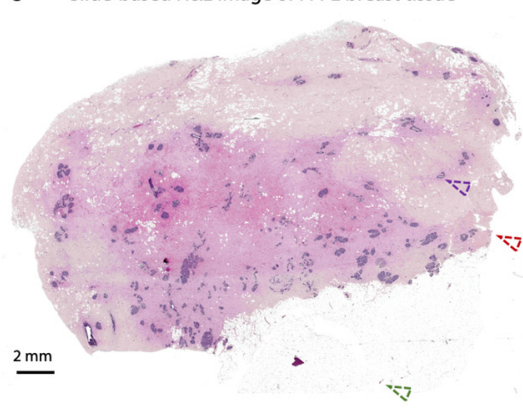

e OTLS microscopy

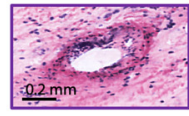

f OTLS microscopy

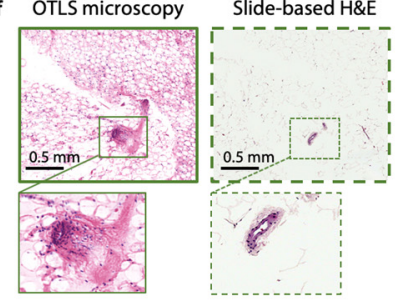

Fig. 5. A fresh breast specimen $(1 \mathrm{~cm}$ by $1 \mathrm{~cm}$ by $0.5 \mathrm{~cm}$, (a)) was first stained with SYBR Gold and ATTO 655 NHS ester followed by surface imaging with an OTLS microscope, (b). After OTLS microscopy, the same piece of tissue was submitted for archival FFPE histology (H\&E), (c). Panels (d), (e) and (f) show benign breast lobules (red arrow), a duct (purple arrow) and a blood vessel within the adipose tissue (green arrow) that were identified from the OTLS surface image, respectively. The corresponding gold-standard H\&E images displaying the same tissue features demonstrate that OTLS microscopy with the SYBR Gold and ATTO 655 NHS ester tissue-staining method can enable rapid and high-quality pathology $\left(1.5 \mathrm{~cm}^{2} /\right.$ minute $)$ of a large surgical specimen surface.

An OTLS surface image of a fresh benign breast specimen $(1 \mathrm{~cm}$ by $1 \mathrm{~cm}$ by $0.5 \mathrm{~cm})($ Fig. $5)$ demonstrates that OTLS microscopy can achieve rapid histologic imaging $\left(1.5 \mathrm{~cm}^{2} /\right.$ minute $)$ of the surface of a large surgical specimen. As shown in Fig. 5, critical breast structures, such as the breast lobules (Fig. 5(d)), a duct (Fig. 5(e)) and a blood vessel within the adipose tissue (Fig. 5(f)), can be identified in the OTLS surface image. The OTLS images in Fig. 5 were compared to corresponding slide-based H\&E images of the same specimen after formalin fixation and paraffin embedding. The quality of our OTLS surface images is similar to that of conventional slide-based H\&E images of FFPE tissues, but with slight differences (see Discussion and conclusion).

Figure 6 displays various microarchitectural features imaged with both OTLS microscopy and conventional slide-based histology. Figure 6(a) shows a benign breast lobule with insets showing individual acini with identifiable lumens. Invasive ductal carcinoma (IDC) with Nottingham grade I (Fig. 6(b)) and Nottingham grade II (Fig. 6(c)) are characterized by tumor 
cells penetrating the basement membrane of the duct and infiltrating the surrounding connective tissues. Figure 6(d) is an example of ductal carcinoma in situ with comedonecrosis.

a
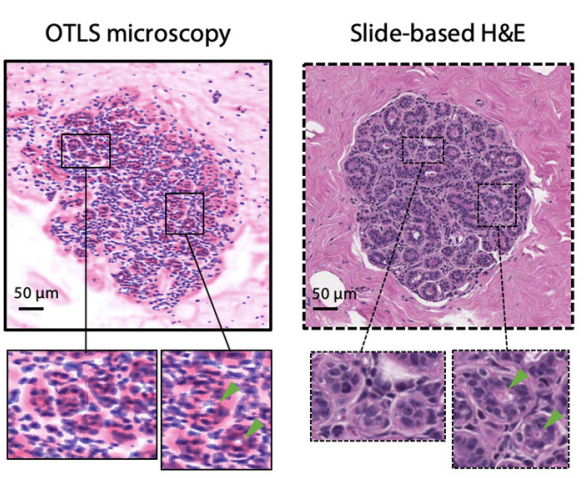

c

Invasive ductal carcinoma ( Nottingham grade II)

OTLS microscopy

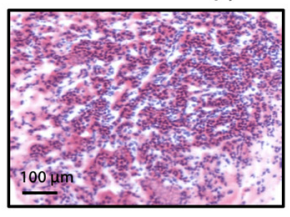

Slide-based H\&E

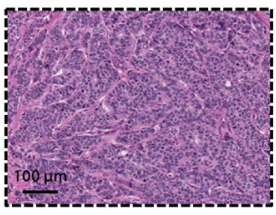

b

Invasive ductal carcinoma

(Nottingham grade I)

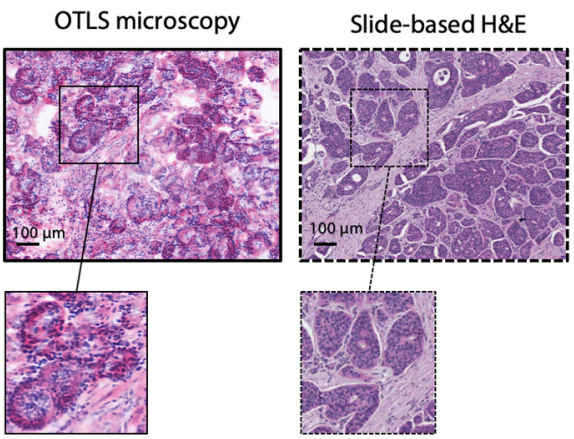

d

Ductal carcinoma in situ with comedonecrosis

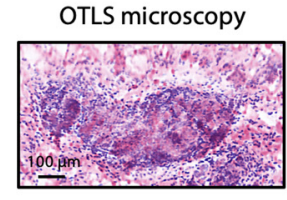

Slide-based H\&E

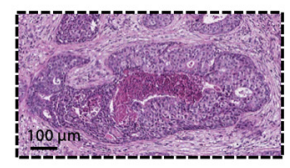

Fig. 6. Various microarchitectural features were identified from the OTLS images, including (a) a benign breast lobule where the inset shows individual acini with identifiable lumens, (b) invasive ductal carcinoma (IDC) with Nottingham grade I and (c) IDC with Nottingham grade II, d) ductal carcinoma in situ with comedonecrosis

Finally, routine H\&E histology and IHC analysis (ER, PR, and HER2 expression) were performed on both control specimens (without OTLS microscopy) and OTLS-microscopyprocessed specimens. Figure 7 shows that the H\&E and IHC results from the control specimens are qualitatively indistinguishable from the OTLS-microscopy-processed specimens, suggesting that our OTLS methods do not interfere with downstream post-operative H\&E histology and IHC analysis. It is worth mentioning that fluorescent histological assays, such as fluorescence in situ hybridization (FISH), have become powerful tools for diagnosing breast cancer $[88,89]$. It is possible that the use of fluorescent dyes (ATTO 655 and SYBR Gold) in our proposed tissue-staining protocol could interfere with downstream FISH assays. However, these fluorescent dyes will most likely be bleached and/or washed away by various harsh chemicals (e.g. xylenes and alcohols) during the histology process for FISH.

\section{Discussion and conclusion}

We previously demonstrated the basic feasibility of utilizing open-top light-sheet (OTLS) microscopy for rapid intraoperative pathology of the surfaces of freshly excised specimens stained with acridine orange (nuclear label) [65]. In this report, we describe an optimized OTLS microscope and image-processing workflow, along with a robust fluorescent analog of H\&E staining, for rapid imaging of breast cancer lumpectomy specimens. Furthermore, we compare the quality of the pseudo-H\&E images generated by our OTLS microscopy system with both frozen-section histology and archival FFPE histology.

As mentioned in the introduction, alternative fresh-tissue microscopy techniques are under development for rapid assessments of surgical specimens, such as confocal microscopy $[33,53-$ 
57], nonlinear microscopy [34,58,59], MUSE [39-43], and SIM [37,60-63]. Impressive imaging speeds have been demonstrated with some of these techniques, such as confocal mosaicking microscopy [57]. However, elaborate tissue flattening and leveling approaches are often needed to keep the tissue surfaces within the narrow focal plane of these 2D microscopy approaches. As mentioned previously, an advantage of OTLS microscopy is the ability to image a thin $3 \mathrm{D}$ volume rapidly, such that irregular and tilted specimen surfaces may be digitally extracted, which has the potential to simplify the imaging process for certain specimens and clinical applications.

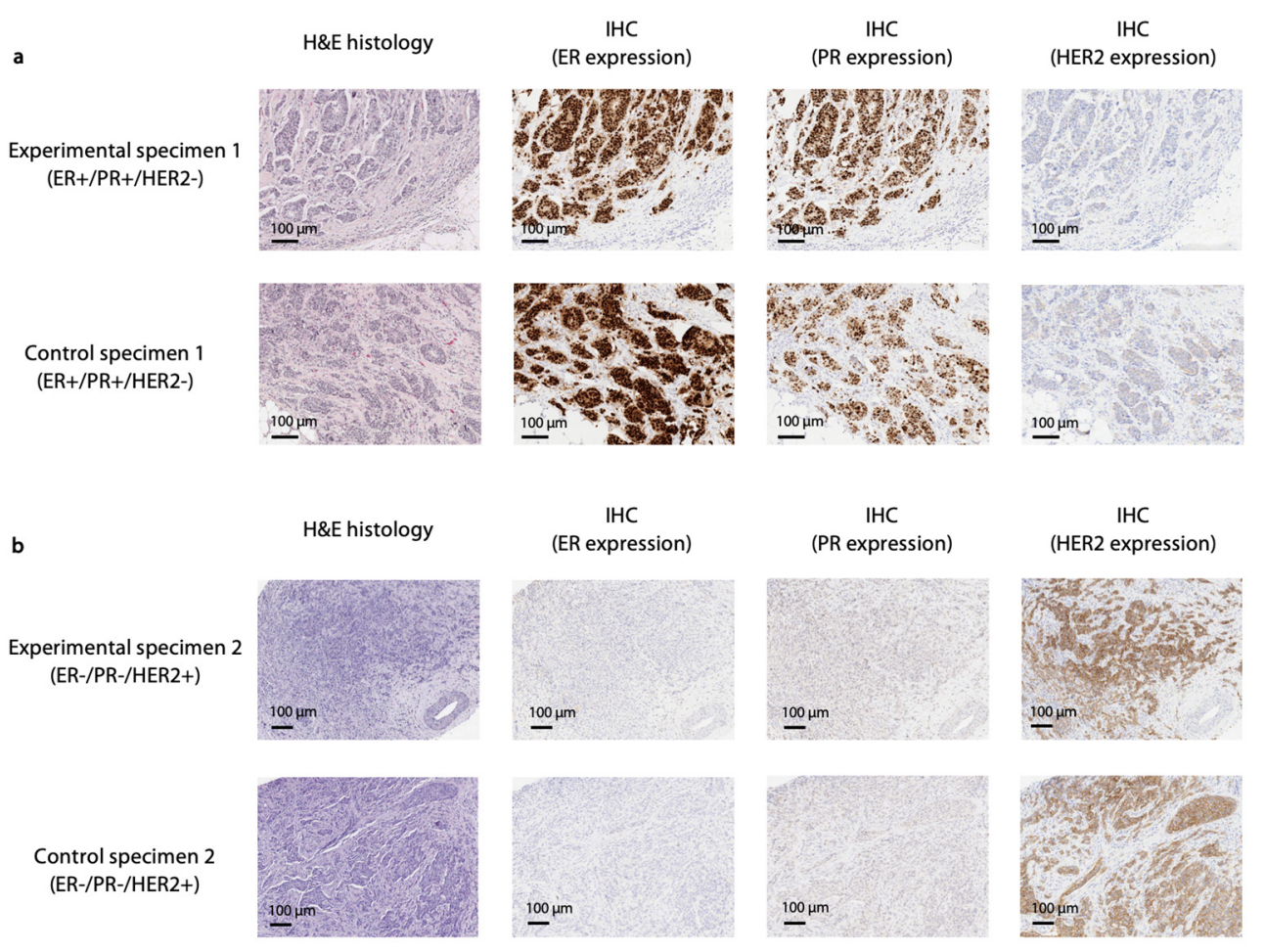

Fig. 7. (a) H\&E histology and (b) IHC results (ER, PR, and HER2 expression) from different control specimens (untouched by OTLS microscopy methods) as compared to those from OTLS microscopy-processed counterparts, showing that the OTLS methods do not interfere with downstream post-operative H\&E histology and IHC analysis.

The OTLS images obtained in this study show relatively good agreement with conventional H\&E histology images, but also exhibit differences. Some of these differences may be attributed to the use of a fluorescent analog of $\mathrm{H} \& \mathrm{E}$ in which the staining characteristics are not chemically identical to conventional H\&E. In addition, the topology and cellular contents at the surfaces of grossly cut tissues are likely to be different when compared to microtomesectioned breast tissues that are formalin fixed and embedded in paraffin (Figs. 5 and 6). For example, grossly cut tissue surfaces are likely to contain few, if any, cells that are physically cut open, and may also show slightly different glandular components, such as the acini shown in Figs. 5(d) and 6(a). In addition, grossly cut specimens may contain tissue debris, irregular or soft surfaces that can deform when being flattened and placed on the microscope sample holder, while FFPE tissues are rigidly embedded in wax and sectioned without deformation. For example, the OTLS images of a duct and a blood vessel shown in Figs. 5(e) and 5(f) appear to be flattened and sheared such that some of the luminal surfaces are exposed. Further, the adipose tissue (Fig. 5(f)) shows stronger staining with our ATTO 655 NHS staining protocol compared to routine $\mathrm{H} \& \mathrm{E}$ staining of tissue sections. We hypothesize that this may be due to: 
(1) ATTO 655 NHS ester covalently binding to cytoplasmic proteins in the fat cells while eosin being weakly bound to the tissue; (2) the 4- $\mu$ m-thick FFPE tissue sections being thinner than the optical-sectioning thickness $(\sim 7 \mu \mathrm{m})$ of the OTLS microscope system utilized in this particular study, which makes the FFPE tissue sections appear lighter in color. Note that OTLS systems with higher resolution have been developed, and may help to mitigate some of these issues.

In this study, we present preliminary results to evaluate the feasibility of translating OTLS microscopy into clinical practice for surgical guidance. Future version of the OTLS microscope should realistically approximate the size of a laser printer. Future clinical systems should also contain an enclosure to protect the optical hardware as well as to reduce ambient light such that the OTLS microscope may be used in a standard pathology lab or surgical facility. In terms of imaging speed, the current two-channel OTLS imaging speed $\left(1.5 \mathrm{~cm}^{2} /\right.$ minute $)$ is limited by the highest frame rate of a state-of-the-art sCMOS camera. As higher-speed detectors become available, imaging speeds may be further improved. In the current system, there is a tradeoff between the height of the imaging volume (number of pixels in the $h$ dimension in Fig. 3 ) acquired by the sCMOS camera and imaging speed. For imaging breast tissues, which are pliable and therefore sit relatively flat on the glass-plate sample holder, a relatively shallow image size of 1000-pixels wide by 128-pixels high is used (64 pixels per channel, which corresponds to $\sim 80 \mu \mathrm{m}$ in spatial dimension), which is sufficient to account for most surface irregularities, specimen tilt, and tissue debris. Data storage will present another challenge for clinical translation. For example, a two-channel OTLS dataset of a $2-\mathrm{cm}$ by $2-\mathrm{cm}$ specimen is $\sim 81.6 \mathrm{~GB}$ large. We are exploring various methods [90] to compress the OTLS datasets without causing significant image degradation. Finally, our current multi-step data acquisition and processing pipeline is not well-suited for routine clinical practice and a simpler consolidated software package with improved efficiency is necessary. Larger-scale clinical studies are needed to fully examine these and other clinical-workflow challenges.

In the future, the spatial resolution of the OTLS system can be increased to provide better imaging of subcellular features, such as nucleoli. However, there will be a trade-off between imaging speed and resolution that may not be desired for certain applications, such as intraoperative guidance. In particular, since post-operative slide-based histopathology can still be utilized after nondestructive OTLS microscopy, the sensitivity and specificity requirements of OTLS microscopy may not be as stringent as what is desired for primary diagnosis and grading.

In summary, we have improved and optimized an OTLS system, along with a reliable and fast two-color staining method (2.5 minutes), to generate H\&E false-colored images of fresh breast tissue surfaces within time frames that are conducive to intraoperative use (1.5 $\mathrm{cm}^{2} /$ minute). Our results provide the first comparison between a pseudo-H\&E OTLS microscopy strategy and both frozen-section and archival FFPE histology. In addition, preliminary results suggest that our nondestructive imaging technology is compatible with downstream archival H\&E histology and IHC analysis, which are currently relied upon for definitive clinical diagnoses. While we have described a number of important technical advances in OTLS microscopy, and have assessed the basic feasibility of our system for rapid pathology of lumpectomy margins, rigorous clinical validation is needed to assess the ability to accurately identify various breast pathologies, such as in situ carcinoma, within intraoperative time frames.

\section{Funding}

National Institutes of Health (P30CA015704, R01CA175391, R01DE023497, F32CA213615); University of Washington Royalty Research Fund (A107248); University of Washington CoMotion Innovation Award; Safeway / Fred Hutchinson Cancer Research Center (Breast Cancer SPORE) developmental award; Prostate Cancer Foundation (Young Investigator 
Award); U.S. Department of Defense (PC170176); National Institute of Diabetes and Digestive and Kidney Diseases (DK076169, DK115255).

\section{Disclosures}

A.K.G., N.P.R., and J.T.C.L. are cofounders and shareholders of LightSpeed Microscopy Inc.

\section{References}

1. C. DeSantis, J. Ma, L. Bryan, and A. Jemal, "Breast cancer statistics, 2013," CA Cancer J. Clin. 64(1), 52-62 (2014).

2. N. Hou and D. Huo, "A trend analysis of breast cancer incidence rates in the United States from 2000 to 2009 shows a recent increase," Breast Cancer Res. Treat. 138(2), 633-641 (2013).

3. B. Fisher, S. Anderson, J. Bryant, R. G. Margolese, M. Deutsch, E. R. Fisher, J. H. Jeong, and N. Wolmark, "Twenty-year follow-up of a randomized trial comparing total mastectomy, lumpectomy, and lumpectomy plus irradiation for the treatment of invasive breast cancer," N. Engl. J. Med. 347(16), 1233-1241 (2002).

4. J. A. Jacobson, D. N. Danforth, K. H. Cowan, T. d'Angelo, S. M. Steinberg, L. Pierce, M. E. Lippman, A. S. Lichter, E. Glatstein, and P. Okunieff, "Ten-year results of a comparison of conservation with mastectomy in the treatment of stage I and II breast cancer," N. Engl. J. Med. 332(14), 907-911 (1995).

5. I. Gage, S. J. Schnitt, A. J. Nixon, B. Silver, A. Recht, S. L. Troyan, T. Eberlein, S. M. Love, R. Gelman, J. R. Harris, and J. L. Connolly, "Pathologic margin involvement and the risk of recurrence in patients treated with breast-conserving therapy," Cancer 78(9), 1921-1928 (1996).

6. K. C. Horst, M. C. Smitt, D. R. Goffinet, and R. W. Carlson, "Predictors of local recurrence after breastconservation therapy," Clin. Breast Cancer 5(6), 425-438 (2005).

7. L. Jacobs, "Positive margins: the challenge continues for breast surgeons," Ann. Surg. Oncol. 15(5), 1271-1272 (2008).

8. J. F. Waljee, E. S. Hu, L. A. Newman, and A. K. Alderman, "Predictors of re-excision among women undergoing breast-conserving surgery for cancer," Ann. Surg. Oncol. 15(5), 1297-1303 (2008).

9. R. Jeevan, D. A. Cromwell, M. Trivella, G. Lawrence, O. Kearins, J. Pereira, C. Sheppard, C. M. Caddy, and J. $H$. van der Meulen, "Reoperation rates after breast conserving surgery for breast cancer among women in England: retrospective study of hospital episode statistics," BMJ 345(jul12 2), e4505 (2012).

10. L. Huo, "A practical approach to grossing breast specimens," Ann. Diagn. Pathol. 15(4), 291-301 (2011).

11. K. Coffey, D. D'Alessio, D. M. Keating, and E. A. Morris, "Second-Opinion Review of Breast Imaging at a Cancer Center: Is It Worthwhile?” AJR Am. J. Roentgenol. 208(6), 1386-1391 (2017).

12. N. Cabioglu, K. K. Hunt, A. A. Sahin, H. M. Kuerer, G. V. Babiera, S. E. Singletary, G. J. Whitman, M. I. Ross, F. C. Ames, B. W. Feig, T. A. Buchholz, and F. Meric-Bernstam, "Role for intraoperative margin assessment in patients undergoing breast-conserving surgery," Ann. Surg. Oncol. 14(4), 1458-1471 (2007).

13. T. M. Bydlon, W. T. Barry, S. A. Kennedy, J. Q. Brown, J. E. Gallagher, L. G. Wilke, J. Geradts, and N. Ramanujam, "Advancing optical imaging for breast margin assessment: an analysis of excisional time, cautery, and patent blue dye on underlying sources of contrast," PLoS One 7(12), e51418 (2012).

14. E. Weinberg, C. Cox, E. Dupont, L. White, M. Ebert, H. Greenberg, N. Diaz, V. Vercel, B. Centeno, A. Cantor, and S. Nicosia, "Local recurrence in lumpectomy patients after imprint cytology margin evaluation," Am. J. Surg. 188(4), 349-354 (2004).

15. A. J. Guidi, J. L. Connolly, J. R. Harris, and S. J. Schnitt, "The relationship between shaved margin and inked margin status in breast excision specimens," Cancer 79(8), 1568-1573 (1997).

16. J. M. Jorns, D. Visscher, M. Sabel, T. Breslin, P. Healy, S. Daignaut, J. L. Myers, and A. J. Wu, "Intraoperative frozen section analysis of margins in breast conserving surgery significantly decreases reoperative rates: oneyear experience at an ambulatory surgical center," Am. J. Clin. Pathol. 138(5), 657-669 (2012).

17. F. H. Barakat, I. Sulaiman, and M. A. Sughayer, "Reliability of frozen section in breast sentinel lymph node examination," Breast Cancer 21(5), 576-582 (2014).

18. R. G. Pleijhuis, M. Graafland, J. de Vries, J. Bart, J. S. de Jong, and G. M. van Dam, "Obtaining adequate surgical margins in breast-conserving therapy for patients with early-stage breast cancer: current modalities and future directions," Ann. Surg. Oncol. 16(10), 2717-2730 (2009).

19. J. C. Cendán, D. Coco, and E. M. Copeland 3rd, "Accuracy of intraoperative frozen-section analysis of breast cancer lumpectomy-bed margins,” J. Am. Coll. Surg. 201(2), 194-198 (2005).

20. T. Osako, R. Nishimura, Y. Nishiyama, Y. Okumura, R. Tashima, M. Nakano, M. Fujisue, Y. Toyozumi, and N. Arima, "Efficacy of intraoperative entire-circumferential frozen section analysis of lumpectomy margins during breast-conserving surgery for breast cancer," Int. J. Clin. Oncol. 20(6), 1093-1101 (2015).

21. S. J. Schnitt and M. Morrow, "Should intraoperative frozen section evaluation of breast lumpectomy margins become routine practice?" Am. J. Clin. Pathol. 138(5), 635-638 (2012)

22. A. J. Creager, J. A. Shaw, P. R. Young, and K. R. Geisinger, "Intraoperative Evaluation of Lumpectomy Margins by Imprint Cytology With Histologic Correlation: a Community Hospital Experience," Arch. Pathol. Lab. Med. 126(7), 846-848 (2002).

23. E. K. Valdes, S. K. Boolbol, J. M. Cohen, and S. M. Feldman, "Intra-operative touch preparation cytology; does it have a role in re-excision lumpectomy?" Ann. Surg. Oncol. 14(3), 1045-1050 (2007). 
24. H. Eggemann, T. Ignatov, A. Beni, S. D. Costa, O. Ortmann, and A. Ignatov, "Intraoperative Ultrasound in the Treatment of Breast Cancer," Geburtshilfe Frauenheilkd. 73(10), 1028-1034 (2013).

25. M. Moschetta, M. Telegrafo, T. Introna, L. Coi, L. Rella, V. Ranieri, A. Cirili, A. A. Stabile Ianora, and G. Angelelli, "Role of specimen US for predicting resection margin status in breast conserving therapy," G. Chir. 36(5), 201-204 (2015).

26. M. Ramos, J. C. Díaz, T. Ramos, R. Ruano, M. Aparicio, M. Sancho, and J. M. González-Orús, "Ultrasoundguided excision combined with intraoperative assessment of gross macroscopic margins decreases the rate of reoperations for non-palpable invasive breast cancer," Breast 22(4), 520-524 (2013).

27. R. A. Graham, M. J. Homer, C. J. Sigler, H. Safaii, C. H. Schmid, D. J. Marchant, and T. J. Smith, "The efficacy of specimen radiography in evaluating the surgical margins of impalpable breast carcinoma," AJR Am. J. Roentgenol. 162(1), 33-36 (1994).

28. W. P. Weber, S. Engelberger, C. T. Viehl, R. Zanetti-Dallenbach, S. Kuster, S. Dirnhofer, D. Wruk, D. Oertli, and W. R. Marti, "Accuracy of frozen section analysis versus specimen radiography during breast-conserving surgery for nonpalpable lesions," World J. Surg. 32(12), 2599-2606 (2008).

29. M. Thill, K. Baumann, and J. Barinoff, "Intraoperative assessment of margins in breast conservative surgery-still in use?” J. Surg. Oncol. 110(1), 15-20 (2014).

30. S. A. Boppart, J. Q. Brown, C. S. Farah, E. Kho, L. Marcu, C. M. Saunders, and H. J. C. M. Sterenborg, "Labelfree optical imaging technologies for rapid translation and use during intraoperative surgical and tumor margin assessment," J. Biomed. Opt. 23(2), 1-10 (2017).

31. F. T. Nguyen, A. M. Zysk, E. J. Chaney, J. G. Kotynek, U. J. Oliphant, F. J. Bellafiore, K. M. Rowland, P. A. Johnson, and S. A. Boppart, "Intraoperative Evaluation of Breast Tumor Margins with Optical Coherence Tomography," Cancer Res. 69(22), 8790-8796 (2009).

32. T. T. W. Wong, R. Zhang, P. Hai, C. Zhang, M. A. Pleitez, R. L. Aft, D. V. Novack, and L. V. Wang, "Fast label-free multilayered histology-like imaging of human breast cancer by photoacoustic microscopy," Sci. Adv. 3(5), e1602168 (2017).

33. E. F. Brachtel, N. B. Johnson, A. E. Huck, T. L. Rice-Stitt, M. G. Vangel, B. L. Smith, G. J. Tearney, and D. Kang, "Spectrally Encoded Confocal Microscopy for Diagnosing Breast Cancer in Excision and Margin Specimens," Lab. Invest. 96(4), 459-467 (2016).

34. Y. K. Tao, D. Shen, Y. Sheikine, O. O. Ahsen, H. H. Wang, D. B. Schmolze, N. B. Johnson, J. S. Brooker, A. E. Cable, J. L. Connolly, and J. G. Fujimoto, "Assessment of breast pathologies using nonlinear microscopy," Proc. Natl. Acad. Sci. U.S.A. 111(43), 15304-15309 (2014).

35. H. Tu, Y. Liu, D. Turchinovich, M. Marjanovic, J. Lyngsø, J. Lægsgaard, E. J. Chaney, Y. Zhao, S. You, W. L. Wilson, B. Xu, M. Dantus, and S. A. Boppart, "Stain-free histopathology by programmable supercontinuum pulses," Nat. Photonics 10(8), 534-540 (2016).

36. D. M. McClatchy, B. W. Maloney, E. J. Rizzo, K. D. Paulsen, W. A. Wells, and B. W. Pogue, "Spatial and Spectral Analysis of in-Situ Tumor-Normal Interfaces in Freshly Resected Lumpectomy Slices using Multispectral Structured Light Imaging," in Biophotonics Congress: Biomedical Optics Congress 2018 (Microscopy/Translational/Brain/OTS), OSA Technical Digest (Optical Society of America, 2018), paper CTu4B.3.

37. T. C. Schlichenmeyer, M. Wang, K. N. Elfer, and J. Q. Brown, "Video-rate structured illumination microscopy for high-throughput imaging of large tissue areas," Biomed. Opt. Express 5(2), 366-377 (2014).

38. M. Wang, D. B. Tulman, A. B. Sholl, H. Z. Kimbrell, S. H. Mandava, K. N. Elfer, S. Luethy, M. M. Maddox, W. Lai, B. R. Lee, and J. Q. Brown, "Gigapixel surface imaging of radical prostatectomy specimens for comprehensive detection of cancer-positive surgical margins using structured illumination microscopy," Sci. Rep. 6(1), 27419 (2016).

39. T. Yoshitake, M. G. Giacomelli, L. M. Quintana, H. Vardeh, L. C. Cahill, B. E. Faulkner-Jones, J. L. Connolly, D. Do, and J. G. Fujimoto, "Rapid histopathological imaging of skin and breast cancer surgical specimens using immersion microscopy with ultraviolet surface excitation,” Sci. Rep. 8(1), 4476 (2018).

40. F. Fereidouni, Z. T. Harmany, M. Tian, A. Todd, J. A. Kintner, J. D. McPherson, A. D. Borowsky, M. Lechpammer, J. Bishop, S. G. Demos, and R. Levenson, "Microscopy with ultraviolet surface excitation for rapid slide-free histology," Nat. Biomed. Eng. 1(12), 957-966 (2017).

41. R. Levenson, F. Fereidouni, Z. Harmany, A. Todd, L. Brandi, and S. Demos, "Microscopy with UV Surface Excitation (MUSE): Rapid, Simple, Slide-Free Histology," in Optics in the Life Sciences Congress, OSA Technical Digest (Optical Society of America, 2017), paper NW3C.5.

42. R. Levenson and F. Fereidouni, "MUSE: A New, Fast, Simple Microscopy Method for Slide-Free Histology and Surface Topography," The FASEB Journal 30,1_supplement, 51.3 (2016).

43. F. Fereidouni, A. D. Mitra, S. Demos, and R. Levenson, "Microscopy with UV Surface Excitation (MUSE) for slide-free histology and pathology imaging," Proc. SPIE 9318, Optical Biopsy XIII: Toward Real-Time Spectroscopic Imaging and Diagnosis, $93180 \mathrm{~F}$ (2015).

44. Y. Wang, S. Kang, A. Khan, G. Ruttner, S. Y. Leigh, M. Murray, S. Abeytunge, G. Peterson, M. Rajadhyaksha, S. Dintzis, S. Javid, and J. T. C. Liu, "Quantitative molecular phenotyping with topically applied SERS nanoparticles for intraoperative guidance of breast cancer lumpectomy," Sci. Rep. 6(1), 21242 (2016).

45. S. C. Davis, S. L. Gibbs, J. R. Gunn, and B. W. Pogue, "Topical dual-stain difference imaging for rapid intraoperative tumor identification in fresh specimens," Opt. Lett. 38(23), 5184-5187 (2013). 
46. S. Kang, Y. W. Wang, X. Xu, E. Navarro, K. M. Tichauer, and J. T. C. Liu, "Microscopic investigation of" topically applied nanoparticles for molecular imaging of fresh tissue surfaces," J. Biophotonics 11(4), e201700246 (2018).

47. Y. W. Wang, N. P. Reder, S. Kang, A. K. Glaser, Q. Yang, M. A. Wall, S. H. Javid, S. M. Dintzis, and J. T. C. Liu, "Raman-Encoded Molecular Imaging with Topically Applied SERS Nanoparticles for Intraoperative Guidance of Lumpectomy," Cancer Res. 77(16), 4506-4516 (2017).

48. C. E. Cox, N. N. Ku, D. S. Reintgen, H. M. Greenberg, S. V. Nicosia, and S. Wangensteen, "Touch preparation cytology of breast lumpectomy margins with histologic correlation," Arch. Surg. 126(4), 490-493 (1991).

49. V. Krishnaswamy, A. M. Laughney, K. D. Paulsen, and B. W. Pogue, "Dark-field scanning in situ spectroscopy platform for broadband imaging of resected tissue," Opt. Lett. 36(10), 1911-1913 (2011).

50. A. M. Laughney, V. Krishnaswamy, E. J. Rizzo, M. C. Schwab, R. J. Barth, B. W. Pogue, K. D. Paulsen, and W. A. Wells, "Scatter spectroscopic imaging distinguishes between breast pathologies in tissues relevant to surgical margin assessment," Clin. Cancer Res. 18(22), 6315-6325 (2012).

51. J. Skoch, A. Dunn, B. T. Hyman, and B. J. Bacskai, "Development of an optical approach for noninvasive imaging of Alzheimer's disease pathology,” J. Biomed. Opt. 10(1), 011007 (2005).

52. V. Venugopal, M. Park, Y. Ashitate, F. Neacsu, F. Kettenring, J. V. Frangioni, S. P. Gangadharan, and S. Gioux, "Design and characterization of an optimized simultaneous color and near-infrared fluorescence rigid endoscopic imaging system," J. Biomed. Opt. 18(12), 126018 (2013).

53. N. Sanai, L. A. Snyder, N. J. Honea, S. W. Coons, J. M. Eschbacher, K. A. Smith, and R. F. Spetzler, "Intraoperative confocal microscopy in the visualization of 5-aminolevulinic acid fluorescence in low-grade gliomas," J. Neurosurg. 115(4), 740-748 (2011).

54. H. Inoue, S. E. Kudo, and A. Shiokawa, "Technology insight: Laser-scanning confocal microscopy and endocytoscopy for cellular observation of the gastrointestinal tract," Nat. Clin. Pract. Gastroenterol. Hepatol. 2(1), 31-37 (2005).

55. J. Eschbacher, N. L. Martirosyan, P. Nakaji, N. Sanai, M. C. Preul, K. A. Smith, S. W. Coons, and R. F. Spetzler, "In vivo intraoperative confocal microscopy for real-time histopathological imaging of brain tumors," J. Neurosurg. 116(4), 854-860 (2012).

56. J. K. Karen, D. S. Gareau, S. W. Dusza, M. Tudisco, M. Rajadhyaksha, and K. S. Nehal, "Detection of basal cell carcinomas in Mohs excisions with fluorescence confocal mosaicing microscopy," Br. J. Dermatol. 160(6), 1242-1250 (2009).

57. S. Abeytunge, B. Larson, G. Peterson, M. Morrow, M. Rajadhyaksha, and M. P. Murray, "Evaluation of breast tissue with confocal strip-mosaicking microscopy: a test approach emulating pathology-like examination," J. Biomed. Opt. 22(3), 034002 (2017).

58. M. G. Giacomelli, T. Yoshitake, L. C. Cahill, H. Vardeh, L. M. Quintana, B. E. Faulkner-Jones, J. Brooker, J. L. Connolly, and J. G. Fujimoto, "Multiscale nonlinear microscopy and widefield white light imaging enables rapid histological imaging of surgical specimen margins," Biomed. Opt. Express 9(5), 2457-2475 (2018).

59. L. C. Cahill, M. G. Giacomelli, T. Yoshitake, H. Vardeh, B. E. Faulkner-Jones, J. L. Connolly, C.-K. Sun, and J. G. Fujimoto, "Rapid virtual hematoxylin and eosin histology of breast tissue specimens using a compact fluorescence nonlinear microscope," Lab. Invest. 98(1), 150-160 (2018).

60. M. Wang, H. Z. Kimbrell, A. B. Sholl, D. B. Tulman, K. N. Elfer, T. C. Schlichenmeyer, B. R. Lee, M. Lacey, and J. Q. Brown, "High-resolution rapid diagnostic imaging of whole prostate biopsies using video-rate fluorescence structured illumination microscopy," Cancer Res. 75(19), 4032-4041 (2015).

61. E. S. Flores, M. Cordova, K. Kose, W. Phillips, A. Rossi, K. Nehal, and M. Rajadhyaksha, "Intraoperative imaging during Mohs surgery with reflectance confocal microscopy: initial clinical experience," J. Biomed. Opt. 20(6), 061103 (2015).

62. S. Abeytunge, Y. Li, B. Larson, G. Peterson, E. Seltzer, R. Toledo-Crow, and M. Rajadhyaksha, "Confocal microscopy with strip mosaicing for rapid imaging over large areas of excised tissue," J. Biomed. Opt. 18(6), 061227 (2013).

63. S. Abeytunge, Y. Li, B. Larson, R. Toledo-Crow, and M. Rajadhyaksha, "Rapid confocal imaging of large areas of excised tissue with strip mosaicing," J. Biomed. Opt. 16(5), 050504 (2011).

64. D. S. Gareau, Y. Li, B. Huang, Z. Eastman, K. S. Nehal, and M. Rajadhyaksha, "Confocal mosaicing microscopy in Mohs skin excisions: feasibility of rapid surgical pathology," J. Biomed. Opt. 13, 054001 (2008).

65. A. K. Glaser, N. P. Reder, Y. Chen, E. F. McCarty, C. Yin, L. Wei, Y. Wang, L. D. True, and J. T. C. Liu, "Light-sheet microscopy for slide-free non-destructive pathology of large clinical specimens," Nat. Biomed. Eng. 1(7), 0084 (2017).

66. J. Huisken, J. Swoger, F. Del Bene, J. Wittbrodt, and E. H. K. Stelzer, "Optical Sectioning Deep Inside Live embryos by Selective Plane Illumination Microscopy," Science 305(5686), 1007-1009 (2004).

67. K. Greger, J. Swoger, and E. H. K. Stelzer, "Basic building units and properties of a fluorescence single plane illumination microscope," Rev. Sci. Instrum. 78(2), 023705 (2007).

68. J. Huisken and D. Y. R. Stainier, "Even fluorescence excitation by multidirectional selective plane illumination microscopy (mSPIM)," Opt. Lett. 32(17), 2608-2610 (2007).

69. F. O. Fahrbach, F. F. Voigt, B. Schmid, F. Helmchen, and J. Huisken, "Rapid 3D light-sheet microscopy with a tunable lens," Opt. Express 21(18), 21010-21026 (2013). 
70. F. O. Fahrbach, V. Gurchenkov, K. Alessandri, P. Nassoy, and A. Rohrbach, "Light-sheet microscopy in thick media using scanned Bessel beams and two-photon fluorescence excitation," Opt. Express 21(11), 13824-13839 (2013).

71. C. J. Engelbrecht, K. Greger, E. G. Reynaud, U. Kržic, J. Colombelli, and E. H. K. Stelzer, "Three-dimensional laser microsurgery in light-sheet based microscopy (SPIM)," Opt. Express 15(10), 6420-6430 (2007).

72. R. McGorty, H. Liu, D. Kamiyama, Z. Dong, S. Guo, and B. Huang, "Open-top selective plane illumination microscope for conventionally mounted specimens," Opt. Express 23(12), 16142-16153 (2015).

73. J. Huisken and D. Y. R. Stainier, "Selective plane illumination microscopy techniques in developmental biology," Development 136(12), 1963-1975 (2009).

74. R. M. Power and J. Huisken, “Intraoperative histology: Lightning 3D histopathology,” Nat. Biomed. Eng. 1(7), 0101 (2017).

75. K. N. Elfer, A. B. Sholl, M. Wang, D. B. Tulman, S. H. Mandava, B. R. Lee, and J. Q. Brown, "DRAQ5 and Eosin ('D\&E') as an Analog to Hematoxylin and Eosin for Rapid Fluorescence Histology of Fresh Tissues," PLoS One 11(10), e0165530 (2016).

76. R. S. Tuma, M. P. Beaudet, X. Jin, L. J. Jones, C. Y. Cheung, S. Yue, and V. L. Singer, "Characterization of SYBR Gold nucleic acid gel stain: a dye optimized for use with 300-nm ultraviolet transilluminators," Anal. Biochem. 268(2), 278-288 (1999).

77. G. T. Hermanson, Bioconjugate Techniques (Third Edition) (Academic Press, 2013).

78. Y. W. Wang, A. Khan, M. Som, D. Wang, Y. Chen, S. Y. Leigh, D. Meza, P. Z. McVeigh, B. C. Wilson, and J. T. C. Liu, "Rapid ratiometric biomarker detection with topically applied SERS nanoparticles," Technology (Singap World Sci) 2(2), 118-132 (2014).

79. Y. Wang, S. Kang, J. D. Doerksen, A. K. Glaser, and J. T. C. Liu, "Surgical Guidance via Multiplexed Molecular Imaging of Fresh Tissues Labeled with SERS-Coded Nanoparticles,” IEEE J. Sel. Top. Quantum Electron. 22(4), 154-164 (2016).

80. Y. W. Wang, J. D. Doerksen, S. Kang, D. Walsh, Q. Yang, D. Hong, and J. T. C. Liu, "Multiplexed Molecular Imaging of Fresh Tissue Surfaces Enabled by Convection-Enhanced Topical Staining with SERS-Coded Nanoparticles," Small 12(40), 5612-5621 (2016).

81. D. Wang, Y. Chen, S. Y. Leigh, H. Haeberle, C. H. Contag, and J. T. C. Liu, "Microscopic Delineation of Medulloblastoma Margins in a Transgenic Mouse Model Using a Topically Applied VEGFR-1 Probe," Transl. Oncol. 5(6), 408-414 (2012).

82. J. T. C. Liu, M. J. Mandella, S. Friedland, R. Soetikno, J. M. Crawford, C. H. Contag, G. S. Kino, and T. D. Wang, "Dual-axes confocal reflectance microscope for distinguishing colonic neoplasia," J. Biomed. Opt. 11(5), 054019 (2006).

83. Y. Chen, W. Xie, A.K. Glaser, N.P. Reder, C. Mao, S.M. Dintzis, J.C. Vaughan and J. T.C. Liu, " OTLS microscopy image shearing code," figshare (2019), https://doi.org/10.6084/m9.figshare.7571861.v4.

84. F. Aguet, D. Van De Ville, and M. Unser, "Model-based 2.5-d deconvolution for extended depth of field in brightfield microscopy," IEEE Trans. Image Process. 17(7), 1144-1153 (2008).

85. Y. Chen, W. Xie, A.K. Glaser, N.P. Reder, C. Mao, S.M. Dintzis, J.C. Vaughan and J. T.C. Liu, " False-coloring code for OTLS microscopy," figshare (2019), https://doi.org/10.6084/m9.figshare.7575692.v2.

86. M. G. Giacomelli, L. Husvogt, H. Vardeh, B. E. Faulkner-Jones, J. Hornegger, J. L. Connolly, and J. G. Fujimoto, "Virtual Hematoxylin and Eosin Transillumination Microscopy Using Epi-Fluorescence Imaging," PLoS One 11(8), e0159337 (2016).

87. S. Preibisch, S. Saalfeld, and P. Tomancak, "Globally optimal stitching of tiled 3D microscopic image acquisitions," Bioinformatics 25(11), 1463-1465 (2009).

88. S. Petroni, L. Caldarola, R. Scamarcio, F. Giotta, A. Latorre, A. Mangia, and G. Simone, "FISH testing of HER2 immunohistochemistry 1+ invasive breast cancer with unfavorable characteristics," Oncol. Lett. 12(5), 3115$3122(2016)$.

89. T. W. Jacobs, A. M. Gown, H. Yaziji, M. J. Barnes, and S. J. Schnitt, "Comparison of fluorescence in situ hybridization and immunohistochemistry for the evaluation of HER-2/neu in breast cancer," J. Clin. Oncol. 17(7), 1974-1982 (1999).

90. B. Balazs, J. Deschamps, M. Albert, J. Ries, and L. Hufnagel, "A real-time compression library for microscopy images," bioRxiv 164624, (2017), doi:10.1101/164624. 